\title{
VISCOUS TRANSONIC SPIRAL AND RADIAL FLOW
}

\author{
M. SICHEL and Y. K. YIN \\ Dept. of Aerospace Eng., the University of Michigan, Ann Arbor (Mich.), U.S.A.
}

\begin{abstract}
Similarity solutions of the viscous transonic equation describing source and source vortex flows have been found. These solutions contain shock-like transitions from the supersonic to the subsonic branch of the corresponding inviscid solutions, while the singularity near the sonic point of the inviscid solutions is shifted to a smaller radius. It is shown that this similarity solution is identical to the transonic viscous compressible source and sink flow solutions of Wu (1955) and Sakurai (1958).
\end{abstract}

\section{§ 1. Introduction}

Exact solutions of the equations of two-dimensional inviscid compressible flow for source and source vortex or spiral flow contain limiting circles at or near the sonic point where the velocity gradient becomes infinite $[1,2,3]$. No solutions exist inside these limiting circles. The transonic flow near these limiting circles, where velocity gradients are large, clearly can no longer be described by an inviscid theory. Application of a viscous transonic theory $[4,5,6,7]$ to the flow in the neighborhood of these limiting circles forms the subject of the present paper.

Two- and three-dimensional source and sink flows of a viscous compressible fluid have previously been investigated by $\mathrm{Wu}[8]$, Sakurai [9], and Levey $[10,11]$. Wu and Sakurai found closed form solutions of the one-dimensional Navier-Stokes equations valid in the region of transonic flow. In the present paper the connection between the general viscous transonic theory and the special solutions of $\mathrm{Wu}$ and Sakurai is shown.

\section{§ 2. The viscous transonic equation}

It has been shown [5] that in two-dimensional viscous transonic 
flow the perturbations to the sonic velocity taken in the $\bar{x}$ direction satisfy the equation

$$
\begin{gathered}
U_{X X}-2 U U_{X}+V_{Y}=0 \\
U_{Y}=V_{X},
\end{gathered}
$$

which has been called the viscous-transonic or $\mathrm{V}-\mathrm{T}$ equation. The dimensionless quantities in (1) and (2) are related to the dimensional coordinates $(\bar{x}, \bar{y})$ and corresponding velocity components $(\bar{u}, \bar{v})$ by

where

$$
\begin{gathered}
X=A \bar{x} / \eta \quad \bar{u} / a^{*}=1+\varepsilon U \\
Y=A \sqrt{\left(\frac{1}{2}\right)(\gamma+1)} \varepsilon^{\frac{1}{2}} \bar{y} / \eta \\
\bar{v} / a^{*}=\varepsilon^{\frac{3}{2}} \sqrt{\left(\frac{1}{2}\right)(\gamma+1)} V,
\end{gathered}
$$

$$
A=\left(\frac{1}{2}\right)(\gamma+1)\left[1+(\gamma-1) / P^{\prime \prime}\right]^{-1} .
$$

The characteristic dimension $\eta$ is taken as $\mu^{* \prime \prime} /\left(\varepsilon a^{*} \rho^{*}\right)$ which is of the order of the thickness of a weak shock wave, where $\mu^{* \prime \prime}, a^{*}$, and $\rho^{*}$ are the longitudinal or compressive viscosity, the speed of sound, and the density, all evaluated where the speed is sonic or critical; $\varepsilon$ is a small parameter proportional to the deviation of $\bar{u} / a^{*}$, i.e. $M^{*}$, from the sonic value of unity. Except for the choice of the characteristic length $\eta$ and the use of the full Navier-Stokes equations the derivation of (1) and (2) is identical to the derivation of the inviscid transonic equation. In fact, as noted previously [5], Eq. (1) reduces to the inviscid transonic equations if the term $U_{X X}$ is deleted.

In the present case it is more convenient to use a dimensionless stream function $\psi$ and potential $\phi$ as independent variables with $w$, the speed, and $\theta$, the streamline angle, as the dependent variables. With $\bar{\phi}$ and $\bar{\psi}$ (barred quantities are dimensional) defined by

it can be shown that

$$
\begin{gathered}
\bar{u}=\bar{\phi}_{\bar{x}} ; \bar{v}=\bar{\phi}_{\tilde{y}} \\
\bar{\rho} \bar{u}=\bar{\psi}_{\bar{y}} ; \bar{\rho} \bar{v}=-\bar{\psi}_{\bar{x}}
\end{gathered}
$$

$$
\begin{gathered}
\phi=\left(\bar{\phi} A / \eta a^{*}\right)=X+\mathrm{O}(\varepsilon) \\
\psi=\varepsilon^{\frac{1}{2}} \bar{\psi} A \sqrt{\left(\frac{1}{2}\right)(\gamma+1)} /\left(\rho^{*} a^{*} \eta\right)=Y+\mathrm{O}\left(\varepsilon^{2}\right)
\end{gathered}
$$

to the same order of approximation as (1) and (2). Defining $\theta$ as 
the streamline angle with respect to the undisturbed sonic velocity $a^{*}$ along the $\bar{x}$-axis we have from (3)

$$
\theta=\tan ^{-1}(\bar{v} / \bar{u}) \approx \varepsilon^{\frac{3}{2}} \sqrt{\left(\frac{1}{2}\right)(\gamma+1)} V .
$$

At the same time since $\bar{u}=\bar{w} \cos \theta$ it follows that

so that $W=U$.

$$
w=\bar{w} / a^{*}=1+\varepsilon W=1+\varepsilon U
$$

Upon eliminating $V$ from (1) and (2) and introducing $\phi$ and $\psi$ as independent variables the V-T equation can be written in the form

$$
W_{\phi \phi \phi}-\left(W^{2}\right)_{\phi \phi}+W_{\psi \psi}=0 .
$$

If a dimensionless angle $\Theta$ is defined by

$$
\theta=\varepsilon^{\frac{3}{3}} \sqrt{\left(\frac{1}{2}\right)(\gamma+1)} \Theta
$$

the irrotationality relation (2) becomes

$$
W_{\psi}=\Theta_{\phi} .
$$

In the inviscid case, that is without the term $W_{\phi \phi \phi}, \mathrm{Eq}$. (8) reduces to the equation considered by Tomotika and Tamada [12] in their study of inviscid transonic nozzle and spiral flow.

\section{§ 3. Similarity solution for spiral flow}

The transformation

$$
W=f(S) ; S=\phi+\lambda \psi
$$

introduced in [12] leads to an exact solution of the inviscid transonic equation which may be interpreted as a spiral flow. The arbitrary parameter $\lambda$ determines the circulation of the source vortex or spiral flow while $S$ is a coordinate in the direction of the radius from the source center. The transformation (10) is also successful in the viscous case and reduces the partial differential equation (8) for $W$ to the ordinary differential equation

$$
f^{\prime \prime \prime}-\left(f^{2}\right)^{\prime \prime}+\lambda^{2} f^{\prime \prime}=0
$$

for the function $f$. Equation (11) is readily integrated twice yielding the Riccati equation

$$
f^{\prime}-f^{2}+\lambda^{2} f=-C_{1} S+C_{2},
$$

where $C_{1}$ and $C_{2}$ are constants of integration, and the minus sign 
ahead of $C_{1}$ is chosen for later convenience. Combination of (9), (10), and (12) yields the expression

$$
\Theta=\lambda f(S)+C_{1} \psi+C_{3}
$$

for the angle $\Theta$, which is valid for both the viscous and inviscid cases; $C_{3}$ is again a constant of integration.

The interpretation of the solution (10) as spiral flow requires further discussion. From the definition of $S$ in (10) and (3) and (6) it follows that the angle between streamlines, $\psi=$ const, and the

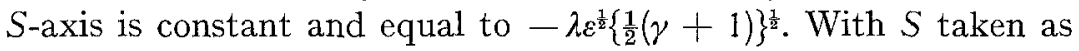
a radial coordinate the streamlines then will be logarithmic spirals described by the equation

$$
\ln \left(\bar{r} \mid \tilde{r}^{*}\right)=-\left(\Omega-\Omega^{*}\right) /\left(\lambda \varepsilon^{\frac{1}{2}} \sqrt{\left(\frac{1}{2}\right)(\gamma+1)}\right) .
$$

The polar angle $\Omega$ is measured with respect to a fixed axis while $\Omega^{*}$ and $\tilde{r}^{*}$ are the particular values of $\bar{r}$ and $\Omega$ at the sonic point of the streamline of interest. With a given streamline chosen for reference the angle $\theta$, on the other hand, is measured with respect to the velocity vector at the sonic point. Fig. 1 shows the distinction between $\theta$ and $\Omega$. It should be mentioned that the solution of (8) presented here describes a family of similar solutions in the same sense as discussed by [13] in connection with viscous transonic nozzle flow. In this latter case $\bar{y} \sim \varepsilon^{-\frac{1}{2}}$ for corresponding streamlines while $\mathrm{d} \bar{y} / \mathrm{d} \bar{x}$, the streamline slope, is $\mathrm{O}\left(\varepsilon^{\frac{8}{2}}\right)$. Rather similarly, in the present case while the angle between the streamline and the radius is $O\left(\varepsilon^{\frac{1}{2}}\right)$ the deviation angle $\theta$ is only $O\left(\varepsilon^{\frac{3}{2}}\right)$.

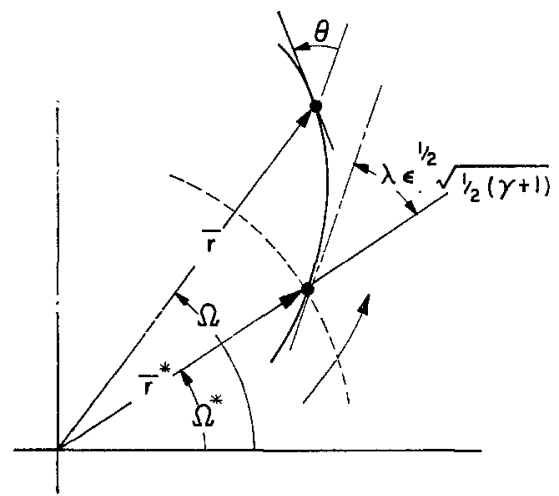

Fig. 1. The geometry for spiral flow. Angles exaggerated for clarity. 


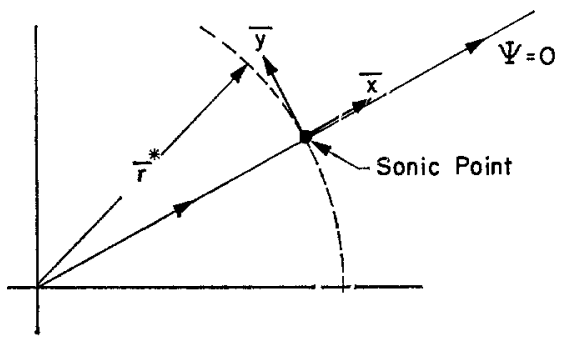

Fig. 2. Radial flow; $X$ is taken in the direction of the $\psi=0$ streamline at the sonic reference point.

Purely radial flow corresponds to $\lambda=0$. Then choosing $\psi=0$ when $\Theta=0$ we have that the constant $C_{3}$ in (13) will be zero so that with $X$ taken in the direction of the $\psi=0$ streamline at the sonic point as shown in Fig. 2, the deviation angle $\theta$ will be

$$
\theta=\varepsilon^{\frac{3}{2}} \sqrt{\left(\frac{1}{2}\right)(\gamma+1)} \Theta=\varepsilon^{\frac{3}{2}} \sqrt{\left(\frac{1}{2}\right)(\gamma+1)} C_{1} Y .
$$

Since $\theta \ll 1$ it follows that in the neighborhood of the sonic reference point, shown in Fig. 2, $\theta \approx \bar{y} / \bar{r}$. Assuming $Y \sim \mathrm{O}(1), C_{1} \sim \mathrm{O}(1)$ it then follows from (15) that for the present theory to be valid the radius $\tilde{r}$, or distance from the source point, must be $O\left(\eta / \varepsilon^{2}\right)$.

Essentially the spiral flow solution describes the flow in the neighborhood of the sonic point on any streamline $\psi=$ const in terms of perturbations to the sonic conditions. With $\psi$ and $\phi$ as coordinates the solution may be interpreted as describing the flow for any arbitrary $\psi$ in a two-dimensional radially symmetric flow. With $X$ and $Y$ as independent variables the solution describes the flow only near that particular streamline with the $X$-axis tangent at the sonic point.

In the case of radial flow it is instructive to develop (12) directly from the Navier-Stokes equations. Then it will be seen that the integration constant $C_{1}$ is related to the source strength. The continuity, momentum, and energy equations for purely radial flow, with $\bar{u}$ the radial velocity, are

$$
\begin{aligned}
\bar{\rho} \bar{u} \bar{r}=\rho^{*} a^{*} r^{*} & \frac{\mathrm{d} \bar{u}}{\mathrm{~d} \bar{r}}+\frac{\mathrm{d} \bar{p}}{\mathrm{~d} \bar{r}}=\frac{\mathrm{d}}{\mathrm{d} \bar{r}}\left[2 \mu \frac{\mathrm{d} \bar{u}}{\mathrm{~d} \bar{r}}+\left(\bar{\mu}^{\prime \prime}-2 \bar{\mu}\right) \times\right. \\
& \left.\times\left(\frac{\mathrm{d} \bar{u}}{\mathrm{~d} \bar{r}}+\frac{\bar{u}}{\bar{r}}\right)\right]+\frac{2 \bar{\mu}}{\bar{r}}\left(\frac{\mathrm{d} \bar{u}}{\mathrm{~d} \bar{r}}-\frac{\bar{u}}{\bar{r}}\right)
\end{aligned}
$$




$$
\begin{aligned}
\rho u C_{\mathrm{p}} & \frac{\mathrm{d} T}{\mathrm{~d} \tilde{r}}-\bar{u} \frac{\mathrm{d} \bar{p}}{\mathrm{~d} \bar{r}}=\frac{\mathrm{d}}{\mathrm{d} \bar{r}}\left(\bar{k} \frac{\mathrm{d} \bar{T}}{\mathrm{~d} \bar{r}}\right)+\frac{2}{3} \bar{u} \times \\
& \times\left[\left(\frac{\mathrm{d} \bar{u}}{\mathrm{~d} \bar{\gamma}}-\frac{\bar{u}}{\bar{\gamma}}\right)^{2}+\left(\frac{\bar{u}}{\bar{r}}\right)^{2}\right]+\left(\bar{\mu}^{\prime \prime}-\frac{4}{3} \mu\right)\left(\frac{\mathrm{d} \bar{u}}{\mathrm{~d} \bar{r}}+\frac{\bar{u}}{\bar{r}}\right)^{2} .
\end{aligned}
$$

To develop approximate equations valid in the transonic regime $\vec{u}, \bar{\rho}$, and $\bar{T}$ are expanded in the form

$$
\bar{L}=L^{*}\left(1+\varepsilon L^{(1)}+\varepsilon^{2} L^{(2)}+\ldots\right)
$$

while $\bar{p}$ is expanded as

$$
\bar{p}=\rho^{*} a^{* 2}\left(\frac{1}{\gamma}+\varepsilon p^{(1)}+\varepsilon^{2} p^{(2)}+\cdots\right),
$$

$\bar{C}_{\mathrm{p}}, \bar{\mu}, \bar{\mu}^{\prime \prime}$, and $\bar{k}$ are assumed constant. If a balance between convection and dissipation is the basic mechanism involved in the flow, it appears reasonable to stretch the $\bar{\gamma}$-coordinate according to

$$
\mathrm{d} \bar{r}=\eta \mathrm{d} \chi
$$

As the region of interest lies near the sonic radius $r^{*}$ it is expedient to express the radial coordinate in the form

$$
r=\frac{\bar{r}}{r^{*}}=1+\chi \frac{\eta}{r^{*}} .
$$

Derivation of an equation for $u^{(1)}$ parallels the original derivations of the $\mathrm{V}-\mathrm{T}$ equation [5] in that substitution of the expansions (19) and (20) and equations (21) and (22) in the conservation equations (16)-(18) yields a set of redundant first order equations and second order equations from which $\rho^{(2)}, u^{(2)}, p^{(2)}$, and $T^{(2)}$ can be eliminated, leading to an equation for $u^{(1)}$. The expansion scheme above will be consistent only if $r^{*} \sim \mathrm{O}\left(\eta / \varepsilon^{2}\right)$, otherwise unbalanced terms occur in the first order equations. This result agrees with the conclusion reached above that $\bar{r} \sim O\left(\eta / \varepsilon^{2}\right)$. Letting $r^{*}=\beta \eta / \varepsilon^{2}$ with $\beta \sim \mathrm{O}(1)$, algebraic reduction leads to the following equation for $u^{(1)}$ :

$$
\left(\frac{\gamma-1}{P r^{\prime \prime}}+1\right) \frac{\mathrm{d}^{2} u^{(1)}}{\mathrm{d} x^{2}}-\frac{1}{2}(\gamma+1) \frac{\mathrm{d}}{\mathrm{d} x}\left(u^{(1) 2}\right)+\frac{1}{\beta}=0 .
$$

Letting $S=A \chi, u^{(1)}=f(S)$, we find that one integration reduces (23) to

$$
f^{\prime}-f^{2}=-S /\left\{\left(\frac{1}{2}\right)(\gamma+1) \beta A\right\}+C_{2} .
$$


Comparing equations (12) and (24) and using the definition of $\beta$ it can now be seen that

$$
C_{1}=2 \eta\left\{\left\{(\gamma+1) r^{*} \varepsilon^{2} A\right\}\right.
$$

so that $C_{1}$ is inversely proportional to $r^{*}$ which in turn is proportional to the source strength. The failure of the present theory when $r^{*} \ll \eta / \varepsilon^{2}$ implies that in this case the balance between convection and dissipation may not be the dominant mechanism so that $\eta$ is no longer the proper characteristic length.

\section{$\S 4$. Closed form solution for $f(S)$}

To actually describe the spiral flow, equation (12) for $f$ must be solved. For later comparison, the inviscid solution for spiral and radial flow will be obtained firstly. Without the viscous term (12) reduces to the algebraic equation

$$
-f^{2}+\lambda^{2} f=-C_{1} S+C_{2} .
$$

The constant $C_{2}$ sets the origin of the $S$ coordinate system and is chosen as zero for convenience. Then the inviscid solution, which

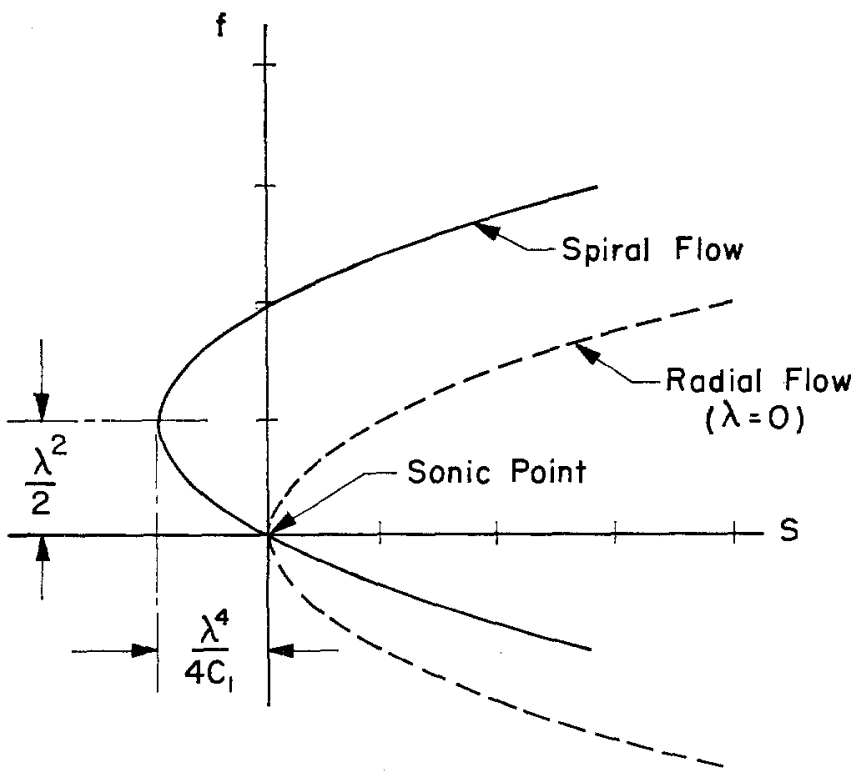

Fig. 3. The inviscid solution (eq. (27)) for spiral flow and radial flow. 
is shown in Fig. 3, becomes

$$
\left(S+\frac{\lambda^{4}}{4 C_{1}}\right)=\frac{1}{C_{1}}\left(f-\frac{\lambda^{2}}{2}\right)^{2} .
$$

The inviscid solution has two branches. Starting at $S=-\lambda^{4} / 4 C_{1}$, $f=\lambda^{2} / 2$, the velocity either continues to accelerate with increasing $S$ or decelerates, reaching the sonic value $f=0$ at $S=0$ and being subsonic for $S>0$. The velocity gradient is infinite at $S=-\lambda^{4} / 4 C_{1}$ while no solution exists for $S<-\lambda^{4} / 4 C_{1}$. Thus, $S=-\lambda^{4} / 4 C_{1}$ represents a limiting circle in the sense discussed in Section 1 above. In the case of radial flow $\lambda=0$ and the sonic and limiting circles coincide so that the solution consists of a subsonic and a supersonic branch. It can be shown that in the transonic regime the inviscid solution (27) is identical to that of [1].

In the viscous case it is convenient to introduce the new variables

$$
\hat{f}=f-\frac{\lambda^{2}}{2} ; \quad \xi=S+\frac{\lambda^{4}}{4 C_{1}},
$$

for then (12), with $C_{2}$ taken equal to zero, becomes

$$
\frac{\mathrm{d} \hat{f}}{\mathrm{~d} \xi}-\hat{f}^{2}=-C_{1} \xi
$$

The Riccati equation (29) is independent of the parameter $\lambda$ so that solutions of (29), upon transforming back to $f$ and $S$, describe both spiral and radial flow.

The additional transformation

$$
\hat{f}=-\frac{1}{T} \frac{\mathrm{d} T}{\mathrm{~d} \xi}
$$

followed by the transformation $\zeta=C_{1}^{1} \xi$ changes (29) to the linear second order equation

$$
T^{\prime \prime}-\zeta T=0
$$

which is Airy's equation, and has the solution [14]

$$
T=C_{4} A_{i}(\zeta)+C_{5} B_{i}(\zeta)
$$

where $A_{i}$ and $B_{i}$ are Airy functions of the first and second kind while $C_{4}$ and $C_{5}$ are constants of integration. From (30) and (32) 
it follows that

$$
\hat{f}=-C_{1}^{\frac{1}{3}} \frac{K A_{i}^{\prime}\left(C_{1}^{\frac{1}{1}} \xi\right)+B_{i}^{\prime}\left(C_{1}^{\frac{1}{3}} \xi\right)}{K A_{i}\left(C_{1}^{\frac{1}{3}} \xi\right)+B_{i}\left(C_{1}^{\frac{1}{3} \xi}\right)},
$$

where the arbitrary constant $K$ has been substituted for the ratio $C_{4} / C_{5}$, while the primes indicate differentiation. It is thus possible to find the solution for spiral and radial viscous transonic flow in closed form. From the asymptotic behavior of the Airy functions [14], it is readily shown that as $\xi \rightarrow \infty$

$$
\hat{f}=\left(f-\frac{\lambda^{2}}{2}\right) \sim-C_{1}^{\frac{1}{2}} \xi^{\frac{1}{2}}=-C_{1}^{\frac{1}{2}}\left(S+\frac{\lambda^{4}}{4 C_{1}}\right)^{\frac{1}{2}} .
$$

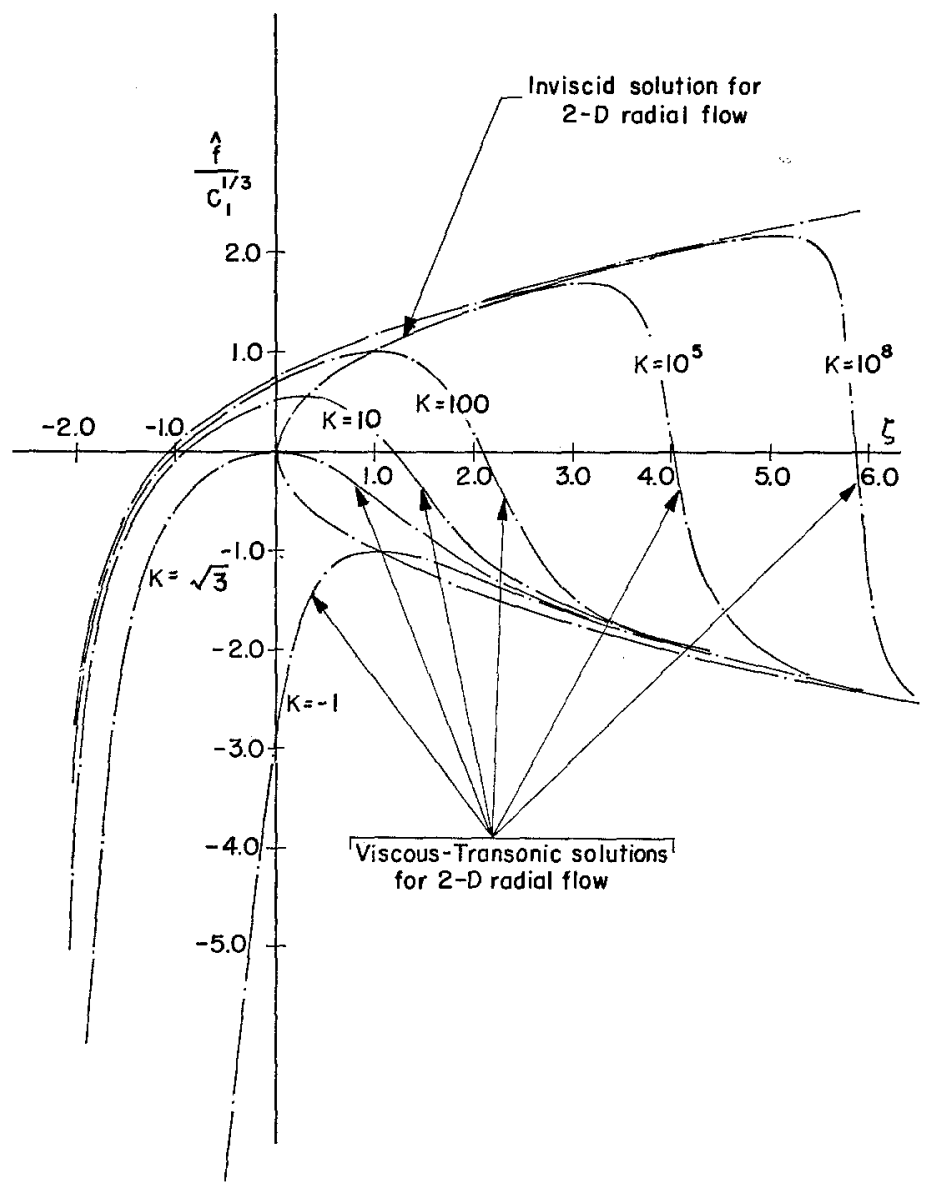

Fig. 4. The function $\tilde{f}$ of the viscous case, for various values of $K$. 
A comparison of (27) and (34) shows the interesting result that as $S \rightarrow \infty$ the viscous solutions all approach the subsonic branch of the inviscid solution.

The behavior of $\hat{f}$ for several values of $K$ is shown in Fig. 4. For large values of $K$ the solution $\hat{f}$ firstly approaches the supersonic branch of the inviscid solution, then after passing through a shocklike compression $\hat{f}$ approaches the subsonic branch of the inviscid solution, as also indicated by the asymptotic solution derived above. From Fig. 4 it is evident that inclusion of the viscous term in the equations for radial flow has eliminated the singular behavior near the sonic point; however, the solution still diverges at some point inside the sonic circle. This can also be seen from the fact that as $\xi \rightarrow-\infty, \hat{f}$ has the asymptotic behavior

$$
\hat{f}=-C_{1}^{\frac{1}{3}}\left(-C_{1}^{\frac{1}{3}} \xi\right)^{\frac{1}{2}} \frac{\left[-(1+K)+(1-K) \tan \left\{\frac{2}{3}\left(-C_{1}^{\frac{1}{2}} \xi\right)^{\frac{3}{2}}+\frac{1}{4} \pi\right\}\right]}{(1-K)+(1+K) \tan \left\{\frac{2}{3}\left(-C_{1}^{\frac{1}{3}} \xi\right)^{\frac{\pi}{2}}+\frac{1}{4} \pi\right\}}
$$

so that $\hat{f}$ has numerous singularities for large negative values of $\xi$.

Fig. 4 also represents the function $f(\zeta)$ for radial flow. From (28) it follows that the behavior of $f(\zeta)$ for spiral flow (i.e. $\lambda \neq 0$ ) can be ascertained from Fig. 4 by simply shifting the origin a distance $\lambda^{2} / 2$ in the minus $\hat{f}$ direction and a distance $\lambda^{4} / 4 C_{1}^{2}$ in the positive $\zeta$ direction.

\section{§ 5. Discussion}

The solution developed above provides another indication that a viscous transonic theory can resolve the singular behavior sometimes encountered in the inviscid theory. As in the case of nozzle flow $[13,15]$ the viscous transonic solutions contain shock-like transitions between the supersonic and subsonic branches of the inviscid solutions. The inviscid singularity at the sonic radius disappears; however, a new singularity occurs at a somewhat smaller radius.

As mentioned above, the present paper is not the first analysis of viscous spiral and source flow. Both $\mathrm{Wu}$ [8] and Sakurai [9] used an approximate form of the Navier-Stokes equation to find source and sink solutions in the transonic flow regime. This approximate equation was developed by using the expansion 


$$
\frac{\bar{u}}{a^{*}}=u=1+\frac{1}{R e^{\frac{1}{3}}} U
$$

with

$$
\ln \frac{\bar{r}}{r^{*}}=\frac{1}{R e^{\frac{2}{3}}} \hat{x}=\ln \left[1+\left(\frac{\bar{r}}{r^{*}}-1\right)\right]
$$

where $R e$ is a Reynolds number defined as $\rho^{*} a^{*} r^{*} / \mu^{*}$. It has already been shown that the V-T solution developed above will be consistent only if

$$
\frac{\eta}{r^{*}}=\frac{\mu^{* \prime \prime}}{\rho^{*} a^{*} r^{*} \varepsilon} \sim \mathrm{O}\left(\varepsilon^{2}\right) .
$$

Since generally $\mu^{* \prime \prime} \mid \mu^{*} \sim \mathrm{O}(1)$ equation (38) implies that $\varepsilon \sim \mathrm{O}\left(R e^{-\frac{1}{2}}\right)$; then if $\tilde{r} / r^{*}-1 \ll 1$, it follows that the expansion and stretching of (19) and (22) and (36) and (37) are equivalent.

The approximate equation obtained by $[8,9]$ is essentially the same as (29) above, and, of course, Wu and Sakurai also found closed form solutions in the transonic case. The significance of the present paper thus lies not in the development of new cylindrical shock solutions, but in showing the connection between the viscous transonic equation (1), which applies to a broad class of twodimensional flows, and the earlier one-dimensional transonic radial flow solutions of $[8,9]$.

A viscous transonic radial flow solution was also obtained by Axford and Newman [16] for the spherically symmetrical radial flow of an ideal gas in a gravitational field. Their interest was to establish the nature of shock transition in solar wind and stellar accretion problems. Axford and Newman's analysis also leads to a Riccati equation for which solutions are obtained in closed form. It is perhaps worth remarking that many problems involving the structure of weak shock waves lead to the solution of a Riccati type equation. The classic example is, of course, the Taylor structure of a weak normal shock wave which is the solution of the equation (Hayes [17])

$$
u^{\prime}-\frac{u^{2}}{2}=\text { constant. }
$$

\section{Acknowledgements}

The authors would like to express their appreciation to the Army 
Research Organization in Durham (N.C.) for their support of this work under Contract DA-31-124-ARO-D-276.

Received 5 March 1968

\section{REFERENCES}

[1] TAYLOR, G. I., 1930 ARCR and M No. 1382, see also The Scientific Papers of G. I. Taylor (ed. G. K. Batchelor), vol. 3, p. 142, Cambridge Un. Press, Cambridge U.K.,

[2] von Mises, R., Mathematical Theory of Compressible Fluid Flow, p. 73, Academic Press, New York 1958.

[3] Oswatitsch, K., Gas Dynamics, p. 258, Academic Press, New York 1956.

[4] Cole, J., Problems in Transonic Flow, Ph.D. thesis, California Institute of Technology, Pasadena (Calif.) 1949.

[5] Sichel, M., Phys. Fluids 6 (1963) 653.

[6] Szaniawski, A., Arch. Mech. Stosow. 15 (1963) 904.

[7] Ryzhov, O. S. and G. M. Shefter, Prik1. Mat. Mekh. 28 (1964) 996.

[8] Wu, T. Y., Quart. Appl. Math. 13 (1955) 393.

[9] Sakurai, A., Quart. J. Mech. Appl. Math. 11 (1958) 274.

[10] Levey, H. C., Quart. Appl. Math. 12 (1954) 25.

[11] Levey, H. C., Quart. Appl. Math. 17 (1959) 77.

[12] Tomotika, S. and K. Tamada, Quart. Appl. Math. 7 (1950) 381.

[13] Sichel, M., J. Fluid Mech. 25 (1966) 769.

[14] Abramowitz, M. and. L. A. Stegun, Handbook of Mathematical Functions, p. 446, U. S. National Bureau of Standards, Washington (D.C.) 1964.

[15] Srchel, M. and Y. K. Yin, J. Fluid Mech. 28 (1967) 513.

[16] Axford, W. L. and R. C. Newman, Astrophys. J. 147 (1967) 230.

[17] Hayes, W. D., Fundamentals of Gas Dynamics, Sec. D., Princeton Un. Press 1958. 